\title{
Peertechz
}
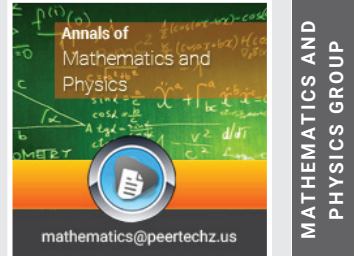

\section{From linear algebra to quantum information}

\section{LW Yu ${ }^{1}$, NL Wang $^{2 \star}$ and S Kanemitsu ${ }^{3}$}

'Department of Physics and Astronomy, University of California, Irvine, 92617, USA

${ }^{2}$ College of Applied Mathematics and Computer Science, Shangluo University, Shangluo, 726000, Shaanxi, P.R. China

${ }^{3}$ Faculty of Engineering, Kyushu Institute of Technology, Kitakyushu 804-08555, Japan
Received: 12 April, 2021

Accepted: 16 July, 2021

Published: 20 July, 2021

*Corresponding authors: Nianliang Wang, Professor, College of Applied Mathematics and Computer Science, Shangluo University, Shangluo, 726000 , Shaanxi, P.R. China, Tel: +86-09142366563;

E-mail:wangnianliangshangluo@aliyun.com

ORCID: https://orcid.org/0000-0003-1980-2268

https://www.peertechzpublications.com

\section{Check for updates}

\section{Abstract}

Anticipating the realization of quantum computers, we propose the most reader-friendly exposition of quantum information and qubits theory. Although the latter lies within framework of linear algebra, it has some flavor of quantum mechanics and it would be easier to get used to special symbols and terminologies. Quantum mechanics is described in the language of functional analysis: the state space (the totality of all states) of a quantum system is a Hilbert space over the complex numbers and all mechanical quantities are taken as Hermite operators. Hence some basics of functional analysis is necessary. We make a smooth transition from linear algebra to functional analysis by comparing the elements in these theories: Hilbert space vs. finite dimensional vector space, Hermite operator vs. linear map given by a Hermite matrix. Then from Newtonian mechanics to quantum mechanics and then to the theory of qubits. We elucidate qubits theory a bit by accommodating it into linear algebra framework under these precursors

\section{Introduction}

Our purpose is to provide basics of quantum information by accommodating it within framework of linear algebra via quantum mechanics and functional analysis in plausibly the most reader-friendly way. This attempt was made in $[1, \mathrm{pp}$. 16-46] and our presentation here is its development with more elucidation. It is usually the case that one takes some basic knowledge for granted without recourse to its source. E.g. the Kronecker product of two matrices is taken as heavensent axiom. It is, however, the matrix associated to the tensor product of two linear maps. Or one uses $C^{n}$ as an $n$-dimensional $\mathrm{C}$-vector space and the underlying result is neglected that an $n$-dimensional $K$ vector space is isomorphic to $K^{n}$, i.e. vector space structure, linear maps, etc. are all ignored. In this paper we make pavement of some of such cracks on the road. We also restore the lost legay by rferring to standard classics on the subject, Chebyshev, Hermite, Dirac, Pauling, et al.

Quantum mechanics is described by the language of functional analysis, Hilbert spaces and linear operators. In the beginning of development, however, infinite matrices used to be used. Cf. e.g. [2]. Also in [3] and [4] infinite matrices are extensively used. Cf. $\S 4$.
In [5] we remarked the following. True that Chen [6] has rather diligently searched for lost references after 1983, especially [7] which is not mentioned in [8] but is the very genesis of Chebyshev-Markov Expansion (CME) and arithmetical Fourier transforms in [9]. It is taken up in [10] as a paper containing infinite matrices in connection with Möbius inversion formula.

More recently [11] treats infinite matrices in another context: "An infinite dimensional vector whose components are Fourier coefficients of an automorphic form is characterized as one which is annihilated by an infinite matrix whose entries are the values of a Bessel function."

Along with development of functional analysis, the use of infinite matrices has been abandoned and replaced totally by linear operators. Since functional analysis may be thought of as an advanced deviation of linear algebra, it may be the most accessible way to grasping quantum mechanics passing from linear algebra to functional analysis and thence to quantum theory. This will be given in $\S 2$.

One of the main possible menace on any Grid system connected to many users will come from using conventional crypto-system. There is a possibility of realization of quantum 
computers which will break many crypto-systems. $\S 4$ is a prelude to quantum information which hopefully will help the readers to recognize the underlying structures.

However, in order to recognize the idea of quantum computers, it is necessary to have some basics in quantum mechanics and for this one needs some basics of mechanics since the former is a modification of Newtonian one. In $\S 3$ we give basics on transition from Newtonian mechanics to quantum mechanics,

Then there are two extremal cases. One is qubits theory and the other extreme is continuous variable quantum mechanics which is the theory of infinite objects and is directly connected to quantum mechanics.

We specify to the theory of qubits (quantum bits) in $\S$ 4. For implementation purpose it is more favorable to have simple structures and qubits are simpler and suitable for implementation as quantum circuits, cf. e.g. [12].

\section{Functional analysis as an advanced deviation of linear algebra}

In this section we make a brief transition from linear algebra to functional analysis, stating different notation and terminologies. There are enormous amount of literature on linear algebra and functional analysis. We refer to [13] or [14] for the former and [15] etc. for the latter.

A vector space $V$ over a field $K$ is an algebraic system in which $V$ is an additive Abelian group with scalar multiplication $\lambda x$ by elements $\lambda$ of $K$, where $x \in V$. The scalar multiplication satisfies standard laws in algebra. It follows that $V$ is the set of all linear combinations with coefficients in $K$. As is always the case with mathematics, given two systems $V, V$ with the same structure, the map is of the most importance that keeps algebraic structure, i.e. $f(x-y)=f(x)-f(y)$ and $\lambda f(x)=f(\lambda x)$. These two conditions are equivalent ot linearity $f(\lambda x+\mu y)=\lambda f(x)+\mu f(y)$ and we call such a map a 1 inear map $f: V \rightarrow V$. Every vector space $V$ has a basis $\left\{u_{i} \mid i \in I\right\}$, which is the smallest set of generators. I.e. every element $x \in V$ may be expressed uniquely as a linear combination $x=\sum_{i \in I} \lambda_{i} u_{i}$ with only finitely many $\lambda_{i}$ 's are non-zero, denoted $V=\oplus_{i \in I} K u_{i}$. If the basis consists of finitely many elements, $V$ is called finite-dimensional and otherwise infinite-dimensional. In the former case, an $\mathrm{n}$-dimensional $\mathrm{K}$-vector space is isomorphic to $\mathrm{K}^{\mathrm{n}}$.

Theorem 2.1 Let

$$
V=\bigoplus_{i=1}^{n} K u_{i}, \quad V^{\prime}=\bigoplus_{j=1}^{m} K v_{j}, \quad V^{\prime \prime}=\underset{k=1}{\bigoplus} K w_{k}
$$

be vector spaces and let $f: V \rightarrow V$ be a linear map. Then we have the basis correspondence formula

$$
f\left(u_{j}\right)=\sum_{i=1}^{m} a_{i j} v_{i}, \quad a_{i j} \in K, \quad 1 \leq j \leq n .
$$

or in row form

$$
\left(f\left(u_{1}\right), \cdots, f\left(u_{n}\right)\right)=\left(v_{1}, \cdots, v_{m}\right) A, \quad A=\left(a_{i j}\right) \in M_{m, n}(K),
$$

Under (3), the linear map $y=f(x)$ is in column form

$$
\left(\begin{array}{c}
y_{1} \\
\vdots \\
y_{m}
\end{array}\right)=\mathrm{y}=f_{A}(\mathrm{x})=A \mathrm{x}=A\left(\begin{array}{c}
x_{1} \\
\vdots \\
x_{n}
\end{array}\right) \text {, }
$$

which means

$$
\sum_{i=1}^{m} y_{i} v_{i}=\sum_{i=1}^{m}\left(\sum_{j=1}^{n} a_{i j} x_{j}\right) v_{i}
$$

and is referred to as the coordinate correspondence formula. The representation matrix of $g \circ f$ is $B A$, where $g: V \rightarrow V$ is a linear map satisfying the same conditions as $f$ with the representation matrix $B$.

The correspondence between a linear map $f$ and a matrix $A$ is one-to-one and a linear map may be treated as $f_{A}$ in (4).

Here matrix product is carried out according to the rule: row $\times$ column. It has chirality and if we express (2) in column vector form, we have ${ }^{t} A$ in (4).

The field $K$ of scalars may be any field, e.g. in coding theory, finite fields are taken. We choose $K$ to be the complex number field $C$.

The inner product (scalar product) of two vectors $a, b$ is defined by

$$
(a, b)={ }^{\mathrm{t}} \mathrm{a} \overline{\mathrm{b}}={ }^{\mathrm{t}} \overline{\mathrm{b}} \mathrm{a}=b a^{*}
$$

This entails non-negativity

$$
(\mathrm{a}, \mathrm{a})=\sum_{i=1}^{n}\left|a_{i}\right|^{2}=|\mathrm{a}|^{2} \geq 0
$$

The inner product satisfies linearity in the first entry

$$
(\mathrm{a}+\mathrm{b}, \mathrm{c})=(\mathrm{a}, \mathrm{c})+(\mathrm{b}, \mathrm{c}), \quad(\mathrm{ca}, \mathrm{b})=c(\mathrm{a}, \mathrm{b})
$$

and because of the complex conjugation property

$$
(b, a)=\overline{(a, b)}
$$

it satisfies modified linearity

$$
(\mathrm{a}, c \mathrm{~b})=\bar{c}(\mathrm{a}, \mathrm{b}), \quad(\mathrm{a}+\mathrm{b}, \mathrm{c})=(\mathrm{a}, \mathrm{c})+(\mathrm{b}, \mathrm{c}) .
$$

Definition 1: The inner product on a $C$-vector space $V$ is defined as a map $V \times V \rightarrow C$ satisfying the non-negativity (7), linearity in the first entry (8), complex conjugation property (9) and linearity in the second entry (10). A C-vector space is called an inner product space if there is defined an inner product. In this case, the norm is defined by (7), i.e.

$$
\|\mathrm{a}\|=\sqrt{(\mathrm{a}, \mathrm{a})} .
$$

We may speak of orthogonality of vectors. Two vectors $a \neq 0, b \neq 0$ are said to be orthogonal if $(a, b)=0$ as suggested by the cosine theorem. Given an orthogonal system (OS) we may normalize it to be an orthonormal system (ONS) by the Gram-Schmidt process. If a basis of a vector space is an ONS, it 
is called an Orthonormal Basis (ONB). $C^{n}$

Lemma 2.1: (i) For a degree K matrix A with complex numbers entries, the following conditions are equivalent.

(a) $A$ is a normal matrix $\left(A A^{*}=A^{*} A\right)$ and all eigenvalues of $A$ are real numbers.

(b) A can be diagonalized by a unitary matrix $U \in U_{k}(\mathbb{C})$ as $U^{-1} A U=\left(\begin{array}{ccc}\lambda_{1} & & O \\ & \ddots & \\ O & & \lambda_{k}\end{array}\right)=D$,

where $\lambda_{i}$ are real.

(c) $A$ is a Hermite matrix.

(ii) Suppose a Hermite matrix $A$ of degree $k$ is diagonalized by a unitary matrix $U$ as (12). Then

$$
e^{A}=\sum_{n=0}^{\infty} \frac{1}{n !} A^{n}=U\left(\begin{array}{ccc}
e^{\lambda_{1}} & & \\
& \ddots & \\
& & e^{\lambda_{k}}
\end{array}\right) U^{-1} .
$$

In what follows we assume the coefficient matrix $A=\left(a_{i j}\right)_{1 \leq i, j \leq k}$ has constant entries. We consider the homogeneous system of DEs $\left(x_{j}=x_{j}(t)\right.$

$$
\frac{\mathrm{d}}{\mathrm{d} t}\left(\begin{array}{c}
x_{1} \\
\vdots \\
x_{k}
\end{array}\right)=A\left(\begin{array}{c}
x_{1} \\
\vdots \\
x_{k}
\end{array}\right)
$$

in the form

$$
\frac{\mathrm{d}}{\mathrm{d} t} \mathrm{x}(t)=A \mathrm{x}(t)
$$

by writing

$$
\mathrm{x}=\mathrm{x}(t){ }^{t}\left(x_{1}(t), \cdots, x_{k}(t)\right)
$$

as a generalization of the $\mathrm{DE}$ for the first-order reaction ( $a$ is a constant)

$$
\frac{\mathrm{d}}{\mathrm{d} t} x(t)=\operatorname{ax}(t)
$$

It is weell-known that the solution to (15) is

$$
x(y)=e^{a t} x(0),
$$

so that the system obeying (15) is an exponential phenomenon. This will be applied very often in what follows.

We may also treat the linear DE of degree $k$

$$
\frac{\mathrm{d}^{k} x}{\mathrm{~d} t^{k}}+a_{1} \frac{\mathrm{d}^{k-1} x}{\mathrm{~d} t^{k-1}}+\cdots+a_{k-1} x=0
$$

as a special case of (14) with

$$
\mathrm{x}=\left(\begin{array}{c}
x(t) \\
\vdots \\
x^{(k-1)}(t)
\end{array}\right)
$$

and

$$
A=\left(\begin{array}{ccccc}
0 & 1 & 0 & \cdots & 0 \\
0 & 0 & 1 & \cdots & 0 \\
& & \cdots & & \\
-a_{k-1} & & \cdots & & -a_{1}
\end{array}\right) .
$$

For differentiation of (18) gives $\frac{\mathrm{d}}{\mathrm{d} t} \mathrm{x}(t)={ }^{t}\left(x^{\prime}, \cdots, x^{(k)}\right)$ and the last entry is $-a_{k-1} x-\cdots-a_{1} \frac{\mathrm{d}^{k-1} x}{\mathrm{~d} t^{k-1}}$ by (17) and so (17) amounts
to (14).

Theorem 2.2: Suppose A is a square matrix of degree $k$ which is diagonalizable as in (12). Then the solution of the system of differential equations (14) under the initial condition $\mathrm{x}(0)={ }^{t}\left(x_{1}(0), \cdots, x_{n}(0)\right)$ is given by

$$
\mathrm{x}(t)=e^{A t} \mathrm{x}(0)
$$

A Hilbert space $H$ is an inner product vector spaces over $C$ which is complete with respect to the norm $\|$.$\| , induced by the$ scalar product (,) (as in (11) ). Here completeness means that every Cauchy sequence is convergent which enables analysis to be conducted on $H$. The elements of $H$ are usually complexvalued functions defined on a certain domain. A typical example is $L^{2}(a, b)$ the Hilbert space of square integrable function on the interval $(a, b)$. Thus we call a linear map a linear operator (or an additive operator) which sends a function to another object. Hilbert spaces come in quantum mechanics as the state space (totality of all states) of a quantum system and all (quantum) mechanical quantities are expressed as Hermitian operators (or self-adjoint operators), i.e. linear operators $T: H \rightarrow H$ satisfying $T^{*}=T$, where $T^{*}$ means the conjugate operator, i.e.

$$
\left(T \Psi_{1}, \Psi_{2}\right)=\left(\Psi_{1}, T^{*} \Psi_{2}\right)
$$

Let $\{E(\lambda)\}$ be a resolution of the identity, i.e. each $E(\lambda)$ is a projector having the properties in Proposition 4.1 and they satisfy certain other conditions.

Theorem 2.3: Let $\{E(\lambda)\}$ be a resolution of the identity. Then for a real-valued continuous function $\emptyset(x)$, we have

$$
(T x, y)=\int_{-\infty}^{\infty} \phi(\lambda) \mathrm{d}(E(\lambda) x, y)
$$

defines the self-adjoint operator $\mathrm{T}$ with domain of definition $H$ satisfying $T E(\lambda) \supset E(\lambda) T$. In particular for $\emptyset(\lambda)=\lambda$

$$
(T x, y)=\int_{-\infty}^{\infty} \lambda \mathrm{d}(E(\lambda) x, y)
$$

defines the self-adjoint operator $\mathrm{T}$ with domain of definition $H$. abbreviated as

$$
T=\int_{-\infty}^{\infty} \lambda \mathrm{d} E(\lambda)
$$

called the spectral decomposition of $T$. Here integrals are Stieltjes integrals and reduce to sums for discrete $\{\lambda\}$.

Definition 2: Let $\mathrm{T}$ be a Hermitian operator with dense domain of definition. For a complex number $\lambda$ let 


$$
T_{\lambda}=T-\lambda I
$$

and consider its inverse. $\lambda$ belongs to the spectra $S(T)$ of $T$ if there is no continuous inverse operator $T_{\lambda}^{-1}$ such that $\overline{D\left(T_{\lambda}^{-1}\right)}=\mathcal{H} . S(T)$ is divided into two subsets:

$$
S(T)=P(T) \cup C(T),
$$

where $P(T)$ is the point spectra and $C(T)$ is the continuous spectra. (In general there is residual spectra), defined respectively as follows.

- $\lambda \in P(T)$ means $D\left(T_{\lambda}^{-1}\right)=\varnothing$, i.e. the inverse operator $T_{\lambda}^{-1}$ does not exist.

- $\lambda \in C(T)$ means $\overline{D\left(T_{\lambda}^{-1}\right)}=H$ but $T_{\lambda}^{-1}$ is not continuous.

Theorem 2.4: That $\lambda \in P(T)$ is equivalent to the fact that the equation $T x=\lambda x$ has a non-trivial solution. If this is the case, $\lambda$ is called the eigenvalue of $\mathrm{T}$ and $x \neq 0$ the eigenvetor of $\mathrm{T}$.

The set of all eigenvectors and the zero vector forms a subspace $E_{T}(\lambda)$ of $H$ called the eigenspace of $T$.

For a Hermitian operator $T=\int \lambda \mathrm{d} E(\lambda)$ we have

- $S(T) \subset \mathbb{R}$.

- $\lambda \notin S(T)$ is equivalent to $E\left(\lambda_{1}\right) \neq E\left(\lambda_{2}\right)$ for some open interval $\left(\lambda_{1}, \lambda_{2}\right)$ containing $\lambda$.

- $\lambda \in P(T) \quad$ is equivalent to $E(\lambda) \neq E(\lambda-0)$ and $E_{T}(\lambda)=\operatorname{I} m(E(\lambda)-E(\lambda-0))$

- $\lambda \in C(T)$ is equivalent to $E(\lambda)=E(\lambda-0)$ and for any open interval $\left(\lambda_{1}, \lambda_{2}\right)$ contaning $\lambda$ we have $E\left(\lambda_{1}\right) \neq E\left(\lambda_{2}\right)$.

For a compact operator (or a completely continuous operator), Theorem 2.3 reduces to the spectral decomposition in the form of a series [16, p. 189](1990),p. 189], whose special case is Theorem 4.2 below.

\section{From classical to quantum mechanics}

In this section we provide a transition from classical to quantum mechanics. We shall use notation in $[17,5.1]$ rather than the commonly used one in quantum mechanics.

We shall first see that taking for granted that every physical quantity $\mathrm{T}$ can be represented by a linear operator, which by abuse of language is denoted by the same letter $T$, Hermitian operators are the ones that are needed. For the expectation value of $T$ is given as the inner product

$$
(\psi, T \psi)=\langle\psi \mid T \psi\rangle=\int \psi^{*} T \psi \mathrm{d} r,
$$

where $(T \psi, \psi)$ indicates the inner product in the vector space $H$ and $r=(x, y, z)$ is the position vector. Since expectation value is the average of the measured real values of a physical quantity, it must be real. For this it suffices that the conjugate operator $\mathrm{T}^{*}$ exists such that $T^{*} \supset T$, which means that $\mathrm{T}^{*}$ is an extension of $T . T$ is then called symmetric. Symmetric operators are not always self-adjoint but physical interpretation requires that operators which represent measurable physical quantities must be self-adjoint, and we use symmetric and Hermitian synonymously so is customary.

For a Hermitian operator T, the eigenvectors $|\psi\rangle$ belonging to its eigenvalue $\lambda(\in \mathbb{R})$ are viewed as the quantum state whose mechanical quantity is equal to $\lambda$.

The Hermitian operator $H$ expressing the total energy of a system is called the Hamiltonian. In most of quantum physics books, the Hamiltonian is denoted $\hat{H}$ but we use the same letter $H$ as with the Hamiltonian function.

The wave function (denoted as a ket vector $|\Psi\rangle=|\Psi(r, t)\rangle$, cf. $\$ 4$ ) is a (vector-valued) function in the position vector $r$ and time $t$ and is to satisfy the (time dependent) Schrödinger equation.

$$
i \hbar \frac{\partial}{\partial t}|\Psi(r, t)\rangle=H|\Psi(r, t)\rangle,
$$

where

$$
\hbar=\frac{h}{2 \pi}
$$

is called the reduced Planck constant, referred to as the Planck constant and where

$$
h=2 \pi \hbar=6.624 \times 10^{-27} \mathrm{erg} \times \mathrm{sec}
$$

is the Planck constant.

More concretely, consider a system of $N$ point particles $r_{j}=\left(x_{j}, y_{j}, z_{j}\right)$ with mass $m_{i}, 1 \leq j \leq N$ moving around a three dimensional space under the influence of forces given as a potential energy $V=V(\mathrm{R}, t)$, where

$$
\mathrm{R}=\left(\mathrm{r}_{1}, \cdots, \mathrm{r}_{N}\right)=\left(x_{1}, \cdots, z_{N}\right) \in \mathbb{R}^{3 N}
$$

is a $3 \mathrm{~N}$-dimensional vector. For this the Hamiltonian $\mathrm{H}$ in (28) reads

$$
H=-\frac{\hbar^{2}}{2} \sum_{j=1}^{N} \frac{1}{m_{j}} \Delta_{j}+V,
$$

where $\Delta_{j}$ is the Laplacian operating on each $r_{j}$ defined by

$$
\Delta_{j}=\frac{\partial^{2}}{\partial x_{j}^{2}}+\frac{\partial^{2}}{\partial y_{j}^{2}}+\frac{\partial^{2}}{\partial z_{j}^{2}} .
$$

Hence the Schrödinger equation (28) amounts to the nonrelativistic Schrödinger equation in position basis

$$
i \hbar \frac{\partial}{\partial t} \Psi(\mathrm{R}, t)=H \Psi(\mathrm{R}, t),
$$

where $\Psi(\mathrm{R}, t)$, called the (position-space) wave function, describes the state of the system at time $t$. In this case, there is a postulate (M. Born) that the probability of the positions 
of $N$ particles lying in a certain region $D \subset \mathbb{R}^{3 N}$ is to be proportional to

$$
\int \cdots \int_{D}|\Psi(\mathrm{R}, t)|^{2} \mathrm{dR} .
$$

For this we must have the probability of the total event is to be $\mathbf{1}$, and a fortiori

$$
\int \cdots \int_{\mathbb{R}^{3 N}|\Psi(\mathrm{R}, t)|^{2} \mathrm{dR}<\infty,}
$$

i.e. the $L^{2}$-space $L^{2}\left(\mathbb{R}^{3 N}\right)$ is the place where the wave functions are to lie.

In the form (31), we may easily check the condition for $H$ to be symmetric. Let $D \subset \mathbb{R}^{3 N}$ be either bounded or equal to the whole set. In the former case, we consider only those wave functions which are 0 -extensions, i.e. $\Psi(\mathrm{R}, t)-0$ if $\mathrm{R} \notin D$ and in the case $D=\mathbb{R}^{3 N}$, we take account that $\Psi(\mathrm{R}, t) \rightarrow 0$ quickly enough as one of the position variables tends to $\infty$ in view of (35). We refer to this as a boundary condition. It suffices to consider the case $\mathrm{R} x$, say. By integration by parts, we have ( $\mathrm{R}^{\prime}$ denoting the $(3 N-1)$-dimensional vector)

$$
\begin{aligned}
& \int \cdots \int_{D} \overline{\Psi_{1}(\mathrm{R}, t)} \frac{\partial^{2}}{\partial^{2} x} \Psi_{2}(\cdots, x, \cdots, t) \mathrm{dR} \\
& =-\int \cdots \int_{\partial D} \frac{\partial}{\partial x} \overline{\Psi_{1}(\mathrm{R}, t)} \frac{\partial}{\partial x} \Psi_{2}(\cdots, x, \cdots, t) \mathrm{dR}{ }^{\prime} \\
& =\int \cdots \int_{D} \frac{\partial^{2}}{\partial^{2} x} \overline{\Psi_{1}(\mathrm{R}, t)} \Psi_{2}(\cdots, x, \cdots, t) \mathrm{dR},
\end{aligned}
$$

or (21) with $T^{*}=T=H$.

We shall explain how the Hamiltonian (31) is deduced from the Hamiltonian function. For a conservative system (in which the potential energy does not depend on time) the Hamiltonian function $H=H(R)$ is the total energy (kinetic and potential) of the system:

$$
H=H(\mathrm{R})=T(\mathrm{p})+V(\mathrm{R})=W .
$$

Here the momentum $p_{k}$ related to the direction $x_{k}$ is $m_{k} \dot{x}_{k}$ , so that

$$
\mathrm{p}=\left(p_{1}, \cdots, p_{N}\right)=\left(m_{1} \dot{x}_{1}, \cdots, m_{N} \dot{x}_{N}\right)
$$

and the kinetic energy $T=T(\mathrm{p})$ is

$$
T=\frac{1}{2} \sum_{k=1}^{n} m_{k}\left(\dot{x}_{k}^{2}+\dot{y}_{k}^{2}+\dot{z}_{k}^{2}\right)=\frac{1}{2} \sum_{k=1}^{n} \frac{1}{m_{k}} p_{k}^{2} .
$$

Substituting (38) into (36), we deduce that

$$
H(\mathrm{R})=\frac{1}{2} \sum_{k=1}^{n} \frac{1}{m_{k}} p_{k}^{2}+V(\mathrm{R})=W .
$$

Making the replacement

$$
p_{k} \leftrightarrow \frac{h}{2 \pi i} \frac{\partial}{\partial x_{k}}=-i \hbar \frac{\partial}{\partial x_{k}}, \quad W \leftrightarrow-\frac{h}{2 \pi i} \frac{\partial}{\partial t}=i \hbar \frac{\partial}{\partial t},
$$

we obtain the Hamiltonian (31).

The Hamiltonian (31) operates on the wave function $\Psi(\mathrm{R}, t)$ as

$$
H \Psi(\mathrm{R}, t)=-\frac{\hbar^{2}}{2} \sum_{j=1}^{N} \frac{1}{m_{j}} \Delta_{j} \Psi(\mathrm{R}, t)+V \Psi(\mathrm{R}, t)=i \hbar \frac{\partial \Psi}{\partial t} .
$$

Hence the wave equation (33) amounts to (41).

In case the boundary conditions can be so chosen that the Hamiltonian is Hermitian, then (41) can be solved using the spectral decomposition. [18, pp. 172-185]:

$$
\Psi(\mathrm{R}, t)=U_{t} \Psi(\mathrm{R}, 0), \quad U_{t}=e^{-\frac{i}{\hbar} H t}
$$

where $e^{B}$ is the exponential function in the bounded operator. Hence this may be thought of as a manifestation of exponential phenomenon. We note that $U_{t}$ is a unitary operator [17, p. 175]. We add a standard characterization [18, p. 41].

Theorem 3.1: For a bounded operator $U$ to be unitary it is necessary and sufficient that

$$
U^{*}=U^{-1} \text {. }
$$

Our aim from here is to solve (41) under the assumption that $\Psi$ is of variables separable type

$$
\Psi(\mathrm{R}, t)=\varphi(t) \psi(\mathrm{R})
$$

and prove Theorem 3.3 generalizing the standard Theorem 3.2. Both of them hold on the assumption that $\psi(\mathrm{R})$ is also of variables separable type. Substituting (44) in (41), we have

$$
\left(-\frac{\hbar^{2}}{2} \sum_{j=1}^{N} \frac{1}{m_{j}} \Delta_{j} \psi(\mathrm{R})+V(\mathrm{R}) \psi(x)\right) \varphi(t)=i \hbar \psi(\mathrm{R}) \frac{\mathrm{d} \varphi(t)}{\mathrm{d} t},
$$

whence dividing both sides by $\varphi(t) \psi(\mathrm{R})$ we have

$$
\frac{1}{\psi(\mathrm{R})}\left(-\frac{\hbar^{2}}{2 m} \frac{\partial^{2} \psi}{\partial x^{2}}+V(\mathrm{R}) \psi(\mathrm{R})\right)=\frac{1}{\varphi(t)}\left(i \hbar \frac{\mathrm{d} \varphi(t)}{\mathrm{d} t}\right) .
$$

Since the left-hand side of (46) is a function in R only and the right-hand side in $t$ only, it follows that (46) must be a constant, say $W$. Hence (46) is equivalent to the system

$$
\left\{\begin{array}{cc}
\frac{\mathrm{d} \varphi(t)}{\mathrm{d} t} & =-\frac{i}{\hbar} W \varphi(t) \\
-\frac{\hbar^{2}}{2} \sum_{j=1}^{N} \frac{1}{m_{j}} \Delta_{j} \psi(\mathrm{R})+V(\mathrm{R}) \psi(\mathrm{R}) & =W \psi(\mathrm{R}) .
\end{array}\right\}
$$

The second equation in (47) is often written as

$$
\sum_{j=1}^{N} \frac{1}{m_{j}} \Delta_{j} \psi(\mathrm{R})+\frac{2}{\hbar^{2}}(W-V) \psi(\mathrm{R})=0
$$


and called the Schrödinger amplitude equation for a conservative system of point particles. $W=W_{n}$ represents the energy of the system at its various stationary states and is called the characteristic energy values or eigenvalues of the wave equation.

Since the first equation in (47) is one for the first-order reaction (15), the solution is given by $e^{-\frac{i}{\hbar} W_{n} t}$. Hence by the principle of superposition, the general solution $\Psi(\mathrm{R}, t)$ of (41) is given by

$$
\Psi(\mathrm{R}, t)=\sum_{n} a_{n} \Psi_{n}(\mathrm{R}, t)=\sum_{n} a_{n} \psi_{n}(\mathrm{R}) e^{-i \frac{W_{n}}{\hbar} t} .
$$

Up here the variable separation condition on $\psi_{n}(\mathrm{R})$ was not assumed. From here we assume that $\psi(\mathrm{R})$ is also of variables separable type, in which case we may separate the components as in $[19$, p. 101] and treat the case $(48)$ for $R=x$.

$$
\frac{\mathrm{d}^{2} \psi}{\mathrm{d} x^{2}}+\frac{2 m}{\hbar^{2}}(W-V) \psi(x)=0 .
$$

Since this is a linear DE of degree 2 with variable coefficients, we apply the Lagrange constant variation method) to find its (approximate) solutions (66). Let

$$
\psi=\psi(x)=e^{q(x)},
$$

say, where

$$
q(x)=\frac{i}{\hbar} \int y \mathrm{~d} x
$$

and $y=y(x)$. Then

$$
\frac{\mathrm{d} \psi}{\mathrm{d} x}=\frac{i}{\hbar} y e^{q(x)}
$$

Hence differentiating again, we obtain

$$
\frac{\mathrm{d}^{2} \psi}{\mathrm{d} x^{2}}=\frac{i}{\hbar}\left(\frac{\mathrm{d} y}{\mathrm{~d} x}+\frac{i}{\hbar} y^{2}\right) e^{q(x)} .
$$

Substituting (54) and (51) into (50) and dividing both sides by $\frac{1}{\hbar^{2}} e^{q(x)}$, we transform (50) into

$$
-\frac{\hbar}{i} \frac{\mathrm{d} y}{\mathrm{~d} x}+y^{2}+2 m(W-V)=0
$$

or

$$
\frac{\hbar}{i} \frac{\mathrm{d} y}{\mathrm{~d} x}=2 m(W-V)-y^{2}=p^{2}-y^{2},
$$

say, where

$$
p=\sqrt{2 m(W-V)}
$$

is the classical expression for the momentum.

The Wentzel-Kramers-Billouin Method (WKB method) consists in giving an approximation to the solution of the wave equation. The first term leads to the result obtained by classical mechanics (Newtonian mechanics), the second term to the old-quantum theory result, and higher terms to corrections which brings in the effects characteristic of the new (quantum) mechanics.

In [19], they apply WKB method by viewing $y$ a priori as a function in $h$ and expand it into the Maclaurin series in $t:=\frac{\hbar}{i}$ :

$$
y=y(x, h)=\sum_{n=0}^{\infty} y_{n} t^{n},
$$

where $y_{j}$ are functions in $x$. We compare the coefficients of (57) and (55) in the form

$$
t \frac{\mathrm{d} y}{\mathrm{~d} x}=t\left(\sum_{n 0}^{\infty} y_{n}^{\prime} t^{n}\right)=p^{2}-y^{2}=p^{2}-\left(y_{0}^{2}+2 y_{0} y_{1} t+\left(y_{1}^{2}+2 y_{0} y_{2}\right) t^{2}+\cdots\right) .
$$

Hence

$$
p^{2}-y_{0}^{2}=0, \quad 2 y_{0} y_{1}=-y_{0}^{\prime}, \quad y_{1}^{2}+2 y_{0} y_{2}=-y_{1}^{\prime}, \cdots
$$

so that

$$
y_{0}=p, \quad y_{1}=\frac{-y_{0}}{2 y_{0}}=\frac{-p}{2 p}, \quad y_{2}=-\frac{1}{2 y_{0}}\left(y_{1}^{\prime}+y_{1}^{2}\right) \cdots .
$$

Since

$$
p^{\prime}=\frac{-2 m V^{\prime}}{2 p},
$$

it follows that

$$
y_{1}=\frac{2 m V^{\prime}}{(2 p)^{2}}=\frac{V^{\prime}}{4(W-V)} .
$$

Substituting the expressions for $y_{1}^{\prime}$ and $y_{1}^{2}$ in the third equality in (60), we find that

$$
\begin{aligned}
& y_{2}=-\frac{1}{2 p} \frac{1}{16(W-V)^{2}}\left(4 V^{\prime \prime}(W-V)+5 V^{\prime 2}\right) \\
& =-\frac{1}{32} \frac{1}{\sqrt{2 m}(W-V)^{5 / 2}}\left(4 V^{\prime \prime}(W-V)+5 V^{\prime 2}\right)
\end{aligned}
$$

Incorporating the coefficients $y_{0}, y_{1}, y_{2}$ from (57), (62) and (63), we have an approximation to (52)

$$
\begin{aligned}
& y=y_{0}+\frac{\hbar}{i} y_{1}+\left(\frac{\hbar}{i}\right)^{2} y_{2}+\cdots=p+\frac{\hbar}{i} \frac{V^{\prime}}{4(W-V)} \\
& -\left(\frac{\hbar}{i}\right)^{2} \frac{1}{32} \frac{1}{\sqrt{2 m}(W-V)^{5 / 2}}\left(4 V^{\prime \prime}(W-V)+5 V^{\prime 2}\right)+\cdots
\end{aligned}
$$
(62)

$$
\int y_{1} \mathrm{~d} x=-\frac{1}{4} \log (W-V)+C
$$

we find an approximation 


$$
\psi=M(W-V)^{-\frac{1}{4}} e^{\int \sqrt{2 m(W-V)} \mathrm{d} x}
$$

with $M$ a constant. The probability distribution function is

$$
\bar{\psi} \psi \approx C(W-V)^{-\frac{1}{2}} \approx \frac{1}{p}
$$

in conformity with the classical result that the probability of finding a particle in a range $d x$ is inversely proportional to the velocity in that range and the velocity is proportional to $p$.

Quantization occurs when one attempts to extend the region for $W$ by an associated condition

$$
\int_{C} y \mathrm{~d} x=2 \pi \hbar h n, \quad n=0,1,2, \cdots,
$$

where the integral is a certain contour integral. The first approximation in (64) amounts to the classical quantum theory condition (68) with $p$ in place of $y$. The second approximation in $(64)$ gives

$$
\int_{C} \mathrm{~d} x=2 \pi \hbar\left(n+\frac{1}{2}\right), \quad n=0,1,2, \cdots .
$$

Lemma 3.1: Writing in (50)

$$
\alpha=\alpha(x)=\frac{1}{\hbar^{2}} p^{2},
$$

where $p$ is defined by (56), we find that (50) amounts to the homogeneous equation for a one-dimensional wave $y(x)$

$$
y^{\prime \prime}+\alpha y=0,
$$

which entails (78).

Corollary 3.1 In the case where $\alpha$ is a constant (we may suppose $\alpha>0$ ), this can be solved e.g. by the method of diagonalization, cf. Theorem 2.2 or by the Laplace transform method and the solution to $(71)$ is

$$
y(x)=y(0) \cos \sqrt{\alpha} x+\frac{y(0)}{\sqrt{\alpha}} \sin \sqrt{\alpha} x=A \sin (\sqrt{\alpha} x+\theta),
$$

say. Further if $y(x)$ has the initial value $y(0)=0$ and satisfies the boundary condition that $y(x)=0$ for $x>a>0$, $a$ being big enough. Then (71) amounts to the one-dimensional Schrödinger equation

$$
-\frac{\hbar}{2 m^{2}} y^{\prime \prime}=E y,
$$

where $E=\frac{\hbar}{2 m^{2}} \alpha$. By continuity, $y(a)=0$. The solution (72)

$$
y(x)=\frac{y^{\prime}(0)}{\sqrt{\alpha}} \sin \sqrt{\alpha} x .
$$

For this to vanish at $x=a$, we must have $\sqrt{\alpha} a=\pi n, n \in \mathbb{N}$ or

$$
\alpha=\left(\frac{\pi n}{a}\right)^{2} .
$$

Hence

$$
E=E_{n}=\frac{\hbar}{2 m^{2}}\left(\frac{\pi n}{a}\right)^{2}
$$

are energy levels and

$$
y_{n}(x)=c \sin \frac{\pi n}{a} x
$$

are eigenfunctions belonging to $E_{n}$.

We are in a position to prove Theorem 3.2.

(71) contains the case of simple harmonic motion of a point particle under the restoring force directly proportional to the displacement $x$ acting on it in the direction opposite to $x$, i.e.

$$
m x^{\prime \prime}=-k x
$$

which is the case (71) with $\alpha=\frac{k}{m}$. It is customary to write $\omega$ for the frequency, i.e. $\alpha=\frac{k}{m}$ or $k=m \omega^{2}$. Since the potential inducing the restoring force on the left-hand side of the above equation is $V(x)=\frac{1}{2} x^{2}$, the Hamiltonian function (39) is

$$
H=H(x)=\frac{1}{2} m p_{x}^{2}+\frac{1}{2} m \omega^{2} x^{2}
$$

Making the replacement (40), we find the Hamiltonianharmonic oscillator

$$
H=-\frac{\hbar^{2}}{2 m} \frac{\mathrm{d}^{2}}{\mathrm{~d} x^{2}}+\frac{1}{2} m \omega^{2} x^{2}
$$

and the Schrödinger equation corresponding to (48) with $W=E$ reads

$$
H \Psi(x)=E \Psi(x)
$$

Introducing the notation

$$
x \leftrightarrow \sqrt{\frac{m \omega}{\hbar}} x, \quad \lambda=\frac{2}{\hbar \omega} E,
$$

(81) amounts to

$$
\left(\frac{\mathrm{d}^{2}}{\mathrm{~d} x^{2}}+x^{2}\right) \Psi(x)=\lambda \Psi(x)
$$

which is (86) below satisfied by Hermite polynomials. The solutions satisfying the boundary condition that $\Psi(x) \rightarrow 0$ as $x \rightarrow \infty$ are possible only for $\lambda=\lambda_{n}$ with $2 n+1, n=0,1, \cdots$ and are proportional to (85).

Invoking the normalization of $\Psi$ we have proved 
Theorem 3.2: Under the assumption of (??), eigenfunctions corresponding to eigenvalues $E_{n}=\left(n+\frac{1}{2}\right) \hbar \omega$ are

$$
\Psi_{n}(x)=\sqrt{\frac{1}{2^{n} n !} \sqrt{\frac{m \omega}{\pi \hbar}}} H_{n}\left(\sqrt{\frac{m \omega}{\hbar} x}\right) e^{-\frac{m \omega}{2 \hbar} x^{2}} .
$$

Cf. e.g. [20]. $\mathrm{DE}$

Here the Hermite polynomial $y=H_{n}(x)$ is a solution of the

$$
\frac{\mathrm{d}^{2} y}{\mathrm{~d} x^{2}}-2 x \frac{\mathrm{d} y}{\mathrm{~d} x}+2 n y=0
$$

and

$$
w=e^{-\frac{1}{2} x^{2}} H_{n}(x)
$$

is a solution of

$$
\frac{\mathrm{d}^{2} w}{\mathrm{~d} x^{2}}+\left(2 n+1-x^{2}\right) w=0 .
$$

Hermite polynomials are one of well-known class of orthogonal polynomials (cf. e.g. [21, II, pp.153-231]) and the class of functions given by (83) forms an ONB (orthonormal basis).

There is an explicit formula ([21, II, p. 193 (9)])

$$
H_{n}(x)=\sum_{m=0}^{[n / 2]} \frac{(-1)^{m}(2 x)^{n-2 m}}{m !(n-2 m) !}
$$

which may be proved by the formula

$$
H_{n}(x)=(-1)^{n} e^{x^{2}} D^{n} e^{-x^{2}}
$$

with $D=\frac{\mathrm{d}}{\mathrm{d} x}$. First a few terms are

$$
H_{0}(x)=1, H_{1}(x)=2 x \cdot H_{2}(x) 4 x^{2}-2, H_{3}(x)=8 x^{3}-12 x, \cdots .
$$

Below we give proofs of some of the properties of Hermite polynomials.

(84) reduces to (86).

Proof. We determine the constants $\alpha$ so that by the change of variable

$$
y=e^{\alpha x^{2}} w
$$

(84) reduces to the one without $\frac{\mathrm{d} w}{\mathrm{~d} x}$. We have

$$
\frac{\mathrm{d} y}{\mathrm{~d} x}=e^{\alpha x^{2}}\left(2 \alpha x w+\frac{\mathrm{d} w}{\mathrm{~d} x}\right)
$$

and

$$
\frac{\mathrm{d}^{2} y}{\mathrm{~d} x^{2}}=e^{\alpha x^{2}}\left(\frac{\mathrm{d}^{2} w}{\mathrm{~d} x^{2}}+4 \alpha x \frac{\mathrm{d} w}{\mathrm{~d} x}+2 \alpha\left(2 \alpha x^{2}+1\right) w\right)
$$

Substituting these and dividing by $e^{\alpha x^{2}}$, we see that (84) reads

$$
\frac{\mathrm{d}^{2} w}{\mathrm{~d} x^{2}}+(4 \alpha-2) \frac{\mathrm{d} w}{\mathrm{~d} x}+\left(4 \alpha(\alpha-1) x^{2}+2 \alpha+2 n\right) w=0 .
$$

We choose $\alpha=\frac{1}{2}$. Then the coefficient of $w$ for this choice $2 n+1-x^{2}$

and (86) follows.

The confluent hypergeometric functions are solutions of the DE

$$
x \frac{\mathrm{d}^{2} y}{\mathrm{~d} x^{2}}+(c-x) \frac{\mathrm{d} y}{\mathrm{~d} x}-a y=0
$$

and one of the solutions is given by $\Psi(a, c ; x)$ [21, II, p. 248].

(91) amounts to Whittaker's standard form

$$
\frac{\mathrm{d}^{2} w}{\mathrm{~d} x^{2}}+\left(-\frac{1}{4}+\frac{\varkappa}{x}+\frac{\frac{1}{4}-\mu^{2}}{x^{2}}\right) w=0
$$

where $a=\frac{1}{2}-\varkappa+\mu, c=1+2 \mu$.

Proof. We determine the constants $\alpha, \beta$ so that by the change of variable

$$
y=x^{\alpha} e^{\beta x} w
$$

(91) reduces to the one without $\frac{\mathrm{d} w}{\mathrm{~d} x}$. We have

$$
\frac{\mathrm{d} y}{\mathrm{~d} x}=x^{-\frac{c}{2}} e^{\frac{1}{2} x}\left(\left(\frac{\alpha}{x}+\beta\right) w+\frac{\mathrm{d} w}{\mathrm{~d} x}\right)
$$

and

$$
\frac{\mathrm{d}^{2} y}{\mathrm{~d} x^{2}}=x e^{\beta x}\left(\frac{\mathrm{d}^{2} w}{\mathrm{~d} x^{2}}+2\left(\frac{\alpha}{x}+\beta\right) \frac{\mathrm{d} w}{\mathrm{~d} x}+\left(\frac{\alpha^{2}-\alpha}{x^{2}}+\frac{2 \alpha \beta}{x}+\beta^{2}\right) w\right)
$$

Substituting these and dividing by $x^{\alpha} e^{\beta x}$, we see that (91) reads

$$
\begin{aligned}
& x \frac{\mathrm{d}^{2} w}{\mathrm{~d} x^{2}}+(2(\alpha+\beta x)+c-x) \frac{\mathrm{d} w}{\mathrm{~d} x} \\
& +\left(\frac{\alpha^{2}-\alpha+c \alpha}{x}+2 \alpha \beta-\alpha+c \beta-a+\beta(\beta-1) x\right) w=0 .
\end{aligned}
$$

Citation: Yu LW, Wang NL, Kanemitsu S (2021) From linear algebra to quantum information. Ann Math Phys 4(1): 032-047. 
For the coefficient of $\frac{\mathrm{d} w}{\mathrm{~d} x}$ to vanish, we choose $\alpha=-\frac{c}{2}$, $\beta=\frac{1}{2}$. Then the coefficient of $w$ for this choice is

$$
\frac{-\frac{c^{2}}{4}+\frac{c}{2}}{x}+\frac{c}{2}-a-\frac{1}{4} x .
$$

Dividing (94) by $x$ leads to (92).

(91) reduces to (84) and (86).

Proof. We write the variable $x$ by $x$ in (91) and put

$$
z=\alpha x^{2}
$$

to obtain

$$
\frac{\mathrm{d} y}{\mathrm{~d} x}=2 \alpha x \frac{\mathrm{d} y}{\mathrm{~d} z}, \quad \frac{\mathrm{d} y}{\mathrm{~d} z}=\frac{1}{2 \alpha x} \frac{\mathrm{d} y}{\mathrm{~d} x} .
$$

Hence

$$
\frac{\mathrm{d}^{2} y}{\mathrm{~d} x^{2}}=2 \alpha \frac{\mathrm{d} y}{\mathrm{~d} z}+2 \alpha x \frac{\mathrm{d}}{\mathrm{d} x} \frac{\mathrm{d} y}{\mathrm{~d} z}=\frac{1}{x} \frac{\mathrm{d} y}{\mathrm{~d} x}+(2 \alpha x)^{2} \frac{\mathrm{d}^{2} y}{\mathrm{~d} z^{2}}
$$

or

$$
\frac{\mathrm{d}^{2} y}{\mathrm{~d} z^{2}}=\frac{1}{4 \alpha^{2} x^{2}}\left(\frac{\mathrm{d}^{2} y}{\mathrm{~d} x^{2}}-\frac{1}{x} \frac{\mathrm{d} y}{\mathrm{~d} z}\right)
$$

Substituting in (91), we deduce that

$$
\frac{\alpha}{4 \alpha^{2}}\left(\frac{\mathrm{d}^{2} y}{\mathrm{~d} x^{2}}-\frac{1}{x} \frac{\mathrm{d} y}{\mathrm{~d} z}\right)+\left(c-\alpha x^{2}\right) \frac{1}{2 \alpha x} \frac{\mathrm{d} y}{\mathrm{~d} x}-a y=0
$$

or

$$
\frac{\mathrm{d}^{2} y}{\mathrm{~d} x^{2}}+\left(c-\frac{\alpha}{2}\right) \frac{1}{2 \alpha x} \frac{\mathrm{d} y}{\mathrm{~d} x}-2 \alpha x \frac{\mathrm{d} y}{\mathrm{~d} x}-4 \alpha a y=0 .
$$

Letting $c=\frac{\alpha}{2} \alpha$, we arrive at

$$
\frac{\mathrm{d}^{2} y}{\mathrm{~d} x^{2}}-2 \alpha x \frac{\mathrm{d} y}{\mathrm{~d} x}-4 \alpha a y=0 .
$$

Hence (96) with $\alpha=1$ (whence $c=\frac{1}{2}$ ) and $a=-\frac{1}{2} n$ amounts to (84).

Proposition 3.1: For the Hermite polynomial (87) we have ([21, II, p.194 (16)]

$$
H_{n}(x)=2^{n} \Psi\left(-\frac{1}{2} n, \frac{1}{2} ; x^{2}\right)
$$

and [21, I, p. $267(32)]$

$$
H_{n}(x)=2^{n} x \Psi\left(\frac{1}{2}-\frac{1}{2} n, \frac{3}{2} ; x^{2}\right) .
$$

amounts to (97).

Proof. Recall the asymptotic formula [21, I, p. 278 (1)]

$$
\Psi(a, c ; x)=\sum_{m=0}^{N}(-1) \frac{m}{m !} x^{-a-m}+O\left(|x|^{-a-N-1}\right),
$$

where

$$
N=0,1,2, \cdots, \quad-\frac{3}{2} \pi<\arg x<\frac{3}{2} \pi, \quad|x| \rightarrow \infty .
$$

Comparing the leading coefficients of (87) and (99), we conclude (97).

To show that (98) amounts to (97) we recall [21, I, p. 267 (6)]

$\Psi(a, c ; x)=x^{1-c} \Psi(a-c+1,2-c: x)$,

(100) with $a=-\frac{n}{2}, c=\frac{1}{2}, x \leftrightarrow x^{2}$ establishes (98).

Using Proposition 3.1, Theorem 3.2 reads

Theorem 3.3 Under the assumption of (??), eigenfunctions corresponding to eigenvalues $E_{n}=\left(n+\frac{1}{2}\right) \hbar \omega$ are

$$
\Psi_{n}(x)=\sqrt{\frac{2^{n}}{n !} \sqrt{\frac{m \omega}{\pi \hbar}}} \Psi\left(-\frac{1}{2} n, \frac{1}{2} ; \frac{m \omega}{\hbar} x\right) e^{-\frac{m \omega}{2 \hbar} x^{2}},
$$

where the left-hand side resp. right-hand side $\Psi$ means the wave function resp. confluent hypergeometric function.

A slightly more general class of functions are that of parabolic cylinder functions, [21, pp. 115-132] (in which the header on p. 131 should read "parabolic cylinder functions").

Finally we state the following extreme case which is important in molecular orbital theory. Cf. [22, pp. 16-22].

Example 3.1: We consider the extreme case where the quantum state $\Psi=\Psi(t)$ varies with time variable $t$ only according to the Schrödinger equation (28), If $H \Psi(t)=E \Psi(t)$ and the eigenvalue $E$ is real called the energy levels of the system, $c f$. the first equation in (47). The solution of this equation is called the stationary state on the ground that its expectation does not change with time. The energy level means the values of the energy which the stationary state can assume. (28) amounts to

$$
\frac{\mathrm{d}}{\mathrm{d} t} \Psi(t)=\frac{-i E}{\hbar} \Psi(t)
$$

and the solution given by (42) amounts to 


$$
\Psi(t)=e^{-\frac{i E t}{\hbar}} \Psi(0)
$$

\section{Quantum information}

Given two wave-functions $\psi_{m}, \psi_{n}$, their inner product $\left(\psi_{m}, \psi_{n}\right)$ is usually defined as in (27) by

$$
\left(\psi_{m}, \psi_{n}\right)=\int \overline{\psi_{m}} \psi_{n} \mathrm{~d} r
$$

We show in (112) below that

$$
\left(\psi_{m}, \psi_{n}\right)=\left\langle\psi_{m} \mid \psi_{n}\right\rangle
$$

in the Dirac notation [23].

Let $\left\{u_{i}\right\}$ be an ONB (e.g. (83) in infinite dimensional case). Then an arbitrary wave-function may be expressed as

$$
\psi_{m}=\sum_{i} c_{i m} u_{i}
$$

where $c_{i m}=\left(\psi_{m}, u_{i}\right) \in \mathbb{C}$ such that

$$
\sum_{i}\left|c_{i m}\right|^{2}=1
$$

We define the ket vector $\left|\psi_{m}\right\rangle$ by

$$
\left|\psi_{m}\right\rangle=\left(\begin{array}{c}
c_{1 m} \\
c_{2 m} \\
\vdots
\end{array}\right)
$$

and the bra vector $\left\langle\psi_{m}\right|$ by

$$
\left\langle\psi_{m}|=| \psi_{m}\right\rangle^{*}=\left(\overline{c_{1 m}}, \overline{c_{2 m}}, \cdots\right),
$$

Cf. Table 1.

Table 1: Notation in linear algebra and quantum theory.

\begin{tabular}{|c|c|c|}
\hline Subject & Lin. alg. & Quantum (ftn'al analysis) \\
\hline system & vector space, matrices & Hilbert space, linear operators \\
\hline complex conj. & $\bar{A}$ & $T^{*}$ \\
\hline transpose & ${ }^{t} A$ & $T^{T}$ \\
\hline conj. transpose & $A^{*}$ & $T^{\dagger}$ \\
\hline Hermitian & $A^{*}=A$ & $T^{\dagger}=T$ \\
\hline unitary & $U^{*}=U^{-1}$ & $U^{\dagger}=U^{-1}$ \\
\hline vector & column $^{\dagger}$ & $\left.k^{\prime}|\psi|\right\rangle$ \\
\hline vector & row ${ }^{\dagger}$ & bra $\langle\psi|\left(=|\psi\rangle^{*}\right)$ \\
\hline inner prod. & $(a, b)=b^{*} a$ & $\left\langle\psi_{1} \mid \psi_{2}\right\rangle=\left|\psi_{1}\right\rangle^{*}\left|\psi_{2}\right\rangle$ \\
\hline
\end{tabular}

Here the scalar product $h \psi 1 \mid \psi 2 i$ is as in (105)
(108) reads for the element $\mu_{i}$ of the ONB,

$$
\left|u_{i}\right\rangle=\left(\begin{array}{c}
0 \\
\vdots \\
0 \\
1 \\
0 \\
\vdots
\end{array}\right)=e_{i},
$$

the $i$ th fundamental unit vector. (107) implies that

$$
\left.\| \psi_{m}\right\rangle \mid=1
$$

Substituting (107) into (104), we find that

$$
\begin{aligned}
& \left(\psi_{m}, \psi_{n}\right)=\sum_{i} \overline{\sum_{j} c^{c}}{ }_{j n}\left(u_{i} u_{j}\right)=\sum_{i} \overline{\sum_{j} \bar{c}^{c}}{ }_{j n} \delta_{i j} \\
& =\sum_{i} \overline{c_{i m} c_{i n}}=\left(\left\langle\psi_{m}|,| \psi_{n}\right\rangle\right),
\end{aligned}
$$

i.e. the matrix product of the bra- and the ket-vectors, which we express as $\left\langle\psi_{m} \mid \psi_{n}\right\rangle$ following Dirac's convention [23, p. 19, 1l. 15-16 from below] of contracting two vertical lines into one. Hence (112) leads to (105). Up here the Hilbert spaces comprising of wave functions is of infinite dimensional and is referred to as continuous-variable quantum information. The ket vectors and matrices are of infinite degree, which are rather classical, as stated in $\S 1$. For modern theory cf. e.g. [24].

In what follows we shall confine ourselves to qubits where the associated Hilbert spaces $H$ are finite dimensional complex vector spaces which we may view as $C^{N}$ (as mentioned in $\S$

2. $N=2^{v}$, cf. (119)). Hence a ket vector is an $N$-dimensional column vector and a Hermitian operator is a Hermite matrix. Since qubits are ( $N$-dimensional) unit vectors, unitary matrices are essential because they are isometries: $|U| \psi\rangle|=\| \psi\rangle \mid$. It will turn out that qubits are generated from single qubits $\phi\rangle, \psi\rangle$ by Kronecker product $\phi\rangle \otimes \psi\rangle=\phi \psi\rangle$ which are then processed by unitary matrices. This is expressed by saying that a qubit $\psi\rangle$ is input into a quantum gate $U$ and output is $U \psi\rangle$.

From here to the end of this section we partly refer to $[12,25]$ some materials are taken from there with more detailed and lucid expoundation using more well-known symbols from linear algebra. It is standard to start from the 2 -dimensional complex vector space $\mathcal{H} \simeq \mathbb{C}^{2}=\mathbb{C e}_{1} \oplus \mathbb{C e}_{2}$ (the totality of all single qubits). It is customary to denote the unit basis vectors as two special states $|0\rangle,|1\rangle$ known as computational basis states. In the notation of (110)

$$
|0\rangle=\left(\begin{array}{l}
1 \\
0
\end{array}\right)=e_{1}, \quad|1\rangle=\left(\begin{array}{l}
0 \\
1
\end{array}\right)=e_{2}
$$

These correspond to the spin-up and spin-down states of a particle such as an electron or a proton. All the elements $a|0\rangle+b|1\rangle,|a|^{2}+|b|^{2}=1$ in $\mathcal{H} \simeq \mathbb{C}^{2}$ are called (single) qubits (quantum bits) or a 1-qubit. When qubits are observed 
(or measured), the value is determined either to be 0 or 1 with probability $|a|^{2}$ and $|b|^{2}$, respectively. Measurement is formally conducted by applying the measurement operators in Definition 6 and the coefficients $|a|^{2}$ and $|b|^{2}$ may be thought of as probabilities (cf. Remark 1).

Single qubits are extended to $v$-qubits by way of tensor products. Cf. e.g. [14,26].

Definition 3: For two free $\mathrm{C}$-modules $M, N$ (i.e. C-vector spaces) with bases $\left\{u_{\mu}\right\},\left\{v_{v}\right\}$, their tensor product $M \otimes N$ is the totality of all linear combinations of $u_{\mu} \otimes v_{v}$, which is again a free $\mathrm{C}$-module. For matrices, we specify the tensor product to be the Kronecker product in Definition 4.

Every state of a quantum system is expressed by a ket vector $\psi\rangle$ which is a wave function and satisfies the Schrödinger equation (28):

$$
i \hbar \frac{\partial}{\partial t}|\psi\rangle=H|\psi\rangle .
$$

If $\psi\rangle$ is only timedependent, then Example 28 gives an explicit expression as an exponential phenomenon:

$$
\left.\psi(t)\rangle=e^{-\frac{i H t}{\hbar}} \psi(0)\right\rangle
$$

which is called the time evlolved state.

The state space of a composite quantum system is the tensor product of the state spaces of the component systems. If $\left|\psi_{j}\right\rangle, 1 \leq j \leq n$ are the states of the component systems $\left(N_{j}\right.$ -qubit), then the joint state of the total system is the Kronecker product $\left|\psi_{1}\right\rangle \otimes \cdots \otimes\left|\psi_{n}\right\rangle$, which is an $N=N_{1} \cdots N_{n}$-qubit in $\mathrm{C}^{\mathrm{N}}$ . For the length of this vector is $\left.\| \psi_{1}\right\rangle\left|\cdots \| \psi_{n}\right\rangle \mid=1$ in view of (124).

Hence 2-qubits are all the unit vectors in the state space $\mathbb{C}^{2} \otimes \mathbb{C}^{2}$. Then we define $v$-qubits inductively as unit vectors in (116) below.

For linear maps $f: M \rightarrow M^{\prime}, g: N \rightarrow N^{\prime}$, there exits a unique linear map denoted $f \otimes g$ such that

$$
(f \otimes g)(x \otimes y)=f(x) \otimes g(y) .
$$

$v$-qubits are all the unit vectors in the Hilbert space (state space)

$$
\mathcal{H} \simeq \underbrace{\mathbb{C}^{2} \otimes \cdots \otimes \mathbb{C}^{2}}_{v}
$$

i.e. they are of the form

$$
a_{1} \mathrm{e}_{1}+\cdots+a_{v} \mathrm{e}_{N}
$$

with normalization

$$
\sum_{k=1}^{N}\left|a_{k}\right|^{2}=1
$$

where

$$
e_{1}=|0 \cdots 0\rangle, \cdots, e_{N}=|1 \cdots 1\rangle
$$

and

$$
N=2^{v} .
$$

E.g. $|0 \cdots 0\rangle=\underbrace{|0\rangle \otimes \cdots \otimes|0\rangle}$ is the Kronecker product of $v$ $|0\rangle$ 's. Since $|0\rangle=\left(\begin{array}{l}1 \\ 0\end{array}\right)$, we have $|0 \cdots 0\rangle={ }^{t}(1,0, \cdots, 0)$ by (123).

Definition 4: For any matrices $A=\left(a_{i j}\right)_{1 \leq i \leq m, 1 \leq j \leq n}$ and $B=\left(b_{k \ell}\right)_{1 \leq k \leq p, 1 \leq \ell \leq q}$ their Kronecker product (or tensor product) $A \otimes B$ is defined by

$$
A \otimes B=\left(\begin{array}{lll}
a_{11} B & \cdots & a_{1 n^{B}} \\
& \cdots & \\
a_{m 1} B & \cdots & a_{m n} B
\end{array}\right)=\left(a_{i j} b_{k \ell}\right)
$$

and this extends linearly in both variables. In particular for two ket vectors

$$
|\varphi\rangle=\left(\begin{array}{c}
c_{1} \\
c_{2} \\
\vdots \\
c_{m}
\end{array}\right) \in \mathbb{C}^{m}, \quad|\psi\rangle=\left(\begin{array}{c}
d_{1} \\
d_{2} \\
\vdots \\
d_{n}
\end{array}\right) \in \mathbb{C}^{n}
$$

their Kronecker product is

$$
|\varphi\rangle \otimes|\psi\rangle=\left(c_{i} d_{j}\right)=\left(\begin{array}{c}
c_{1} d_{1} \\
\vdots \\
c_{1} d_{n} \\
\vdots \\
c_{m} d_{1} \\
\vdots \\
c_{m} d_{n}
\end{array}\right),
$$

which we usually express in the transposed row vector form

$$
{ }^{t}\left(c_{1} d_{1}, \cdots, c_{1} d_{n}, \cdots, c_{m} d_{1}, \cdots, c_{m} d_{n}\right) .
$$

The left-hand side of (122) is abbreviated as:

$|\varphi\rangle|\psi\rangle=|\varphi \psi\rangle=|\varphi\rangle \otimes|\psi\rangle=\left(c_{i} d_{j}\right)$,

This satisfies

$\| \varphi\rangle \otimes|\psi\rangle|=\| \varphi\rangle|||\psi\rangle \mid$.

The outer product $|\psi\rangle\langle\phi|$ is the Kronecker product $|\varphi\rangle^{*} \otimes|\psi\rangle$ given by

$$
|\psi\rangle\langle\varphi|=| \varphi\rangle^{*} \otimes|\psi\rangle=\left(\bar{c}_{1}, \cdots, \bar{c}_{m}\right) \otimes\left(\begin{array}{c}
d_{1} \\
d_{2} \\
\vdots \\
d_{n}
\end{array}\right)=\left(\begin{array}{ccc}
\bar{c}_{1} d_{1} & \cdots & \bar{c}_{m} d_{1} \\
& \cdots & \\
\bar{c}_{1} d_{n} & \cdots & \bar{c}_{m} d_{n}
\end{array}\right),
$$


It is convenient to view it as a linear map $\mathrm{C}^{m} \rightarrow \mathrm{C}^{n}$

$$
|\psi\rangle\langle\varphi|\left(\left|\varphi^{\prime}\right\rangle\right)=|\psi\rangle\left\langle\varphi \mid \varphi^{\prime}\right\rangle=\left\langle\varphi \mid \varphi^{\prime}\right\rangle|\psi\rangle \text {. }
$$

If $\left\{e_{j}\right\}$ be an ONB, then we have

$$
\left|e_{i}\right\rangle\left\langle e_{j}\right|=E_{i j},
$$

where $E_{i j}$ is a matrix unit consisting of all o's except for the ( $i$, j)-entry which is 1 . In particular,

$$
P_{k}=\left|e_{k}\right\rangle\left\langle e_{k}\right|=E_{k k}
$$

is called a projector, cf. [17, p. 173] for a projector in a Hilbert space. They have properties stated in (135).

It is instructive to know the source of the Kronecker product.

Theorem 4.1: Suppose $M, M^{\prime}, N, N^{\prime}$ are f.g. free $K$-modules. Then $M^{*}=\operatorname{Hom}_{J}(M, J)$ denoting the dual of $M$, we have

$$
(M \otimes N)^{*} \cong M^{*} \otimes N^{*}
$$

in general. (129) and

$$
\mathrm{Hom}_{R}\left(M \otimes N, M^{\prime} \otimes N^{\prime}\right) \cong \operatorname{Hom}_{R}\left(M, M^{\prime}\right) \otimes \operatorname{Hom}_{R}\left(N, N^{\prime}\right)
$$

are equivalent. (130) gives a motivation for the tensor product of maps in Definition 4.

Suppose $\quad M=\oplus_{i=1}^{n} K u_{i}, \quad M^{\prime}=\oplus_{j=1}^{n^{\prime}} K u_{j}^{\prime}, \quad N=\oplus_{k=1}^{m} K v_{k}$ , and $N^{\prime}=\oplus_{\ell=1}^{m^{\prime}} R v_{\ell}^{\prime} a$ and that the matrices corresponding to $f=f_{A} \in \operatorname{Hom}_{K}\left(M, M^{\prime}\right)$ and $g=g_{B} \in \operatorname{Hom}_{K}\left(N, N^{\prime}\right)$ are as in Theorem 2.1. Then the matrix corresponding to the tensor product $f \otimes g$ with respect to the bases $\left\{u_{i} \otimes v_{k}\right\},\left\{u_{j}^{\prime} \otimes v_{\ell}^{\prime}\right\}$ is the Kronecker product $A \otimes B$ :

$$
(f \otimes g)\left(u_{i} \otimes v_{k}\right)=\left(u_{j}^{\prime} \otimes v_{\ell}^{\prime}\right) A \otimes B .
$$

Example 4.1: $v$-qubits can represent $2^{v}$ states:

$$
|n\rangle=\mathrm{e}_{n+1},
$$

where the left-hand side means the $n$th member of the sequence of $v$ digits numbers in dyadic expansion arranged in increasing order and the right means the $n$th $2^{v}$-dimensional fundamental unit vector.

E.g. for $N=3$, we have by (123) and (122)

$$
|6\rangle=|110\rangle=|1\rangle|1\rangle|0\rangle=|1\rangle \otimes|1\rangle \otimes|0\rangle
$$

$$
=\left(\begin{array}{l}
0 \\
1
\end{array}\right) \otimes\left(\begin{array}{l}
0 \\
1
\end{array}\right) \otimes\left(\begin{array}{l}
1 \\
0
\end{array}\right)=\left(\begin{array}{l}
0 \\
0 \\
0 \\
1
\end{array}\right) \otimes\left(\begin{array}{l}
1 \\
0
\end{array}\right)={ }^{t}(0,0,0,0,0,0,1,0)=e_{7} .
$$

Here the first notation is the decimal number 6 viewed as its dyadic expansion $2^{2}+1 \cdot 2+0$.

Proposition 4.1: (125) and (126) are equivalent. Let $\left\{e_{j}\right\}$ be an
ONB satisfying $\left\langle e_{i} \mid e_{j}\right\rangle=\delta_{i j}$. Then the completeness condition

$$
\sum_{i=1}^{n}\left|e_{i}\right\rangle\left\langle e_{i}\right|=E .
$$

as well as

$$
P_{k}^{2}=P_{k}, \quad\left\langle P_{k} \varphi_{1} \mid \varphi_{2}\right\rangle=\left\langle\varphi_{1} \mid P_{k} \varphi_{2}\right\rangle, \quad P_{k} P_{\ell}=0 \quad(k \neq \ell)
$$

are satisfied, where $P_{k}$ is the projector defined by (128).

Proof. Let $\left|\varphi^{\prime}\right\rangle=\left(\begin{array}{c}c_{1} \\ 1 \\ c_{2} \\ \vdots \\ c_{m}\end{array}\right)$. Then noting $\left\langle\varphi \mid \varphi^{\prime}\right\rangle=\sum_{i=1}^{m} \bar{c}_{i} c_{i}^{\prime}$, we see the equivalence by multiplying $\left|\varphi^{\prime}\right\rangle$ by the matrix (125).

The second assertion follows from (127). Finally the second equality in (135) holds since both sides are $\left\langle e_{k} \mid \varphi_{1}\right\rangle\left\langle e_{k} \mid \varphi_{2}\right\rangle$.

We prove that the totality of all Kronecker products of single qubits $|a\rangle|b\rangle$ does not exhaust the totality of 2 -qubits states.

Proof. 2 -qubits are of the form

$$
a_{1}|00\rangle+a_{2}|01\rangle+a_{3}|10\rangle+a_{4}|11\rangle
$$

with $\sum_{k=1}^{4}\left|a_{k}\right|^{2}=1$.

On the other hand, by (132),

$$
|a\rangle \otimes|b\rangle=\left(a_{1}|0\rangle+a_{2}|1\rangle\right) \otimes\left(b_{1}|0\rangle+b_{2}|1\rangle\right)={ }^{t}\left(a_{1} b_{1}, a_{1} b_{2}, a_{2} b_{1}, b_{1} b_{2}\right),
$$

which cannot exhaust (136).

$$
\begin{aligned}
& \text { E.g. } \\
& \frac{|00\rangle+|11\rangle}{\sqrt{2}}=t\left(\frac{1}{\sqrt{2}}, 0,0, \frac{1}{\sqrt{2}}\right)
\end{aligned}
$$

cannot be expressed in the form (137).

But since the totality of all $\mathrm{N}$-qubits arising from the Kronecker products of component $N_{j}$-qubits have restrictions on the coefficients, it does not exhaust all $N$-qubits. In qubit theory one says that a state of a composite system which cannot be written as a product of states of its component systems is an entangled state. The state (138) is an entangled state.

The following theorem is the special case of Theorem 2.3 for a compact operator as stated toward the end of $\S 2$.

Theorem 4.2: Let $A$ be a Hermite matrix of degree $n$ with eigenvalues $\lambda_{k}$ and the corresponding eigenvectors $\left|\lambda_{k}\right\rangle, 1 \leq k \leq n$ forming an ONB. Then the spectral decomposition

$$
A=\sum_{k=1}^{n} \lambda_{k} P_{k}=\sum_{k=1}^{n} \lambda_{k}\left|\lambda_{k}\right\rangle\left\langle\lambda_{k}\right|
$$


holds true, where $P_{k}$ is the projector in (128). If $f(z)=\sum_{k=0}^{\infty} a_{k} z^{k}$ is an analytic function with power series convergent in a disc, then

$$
f(A)=\sum_{k=1}^{n} f\left(\lambda_{k}\right)\left|\lambda_{k}\right\rangle\left\langle\lambda_{k}\right|
$$

and in particular,

$$
e^{A}=\sum_{k=1}^{n} e^{\lambda_{k}}\left|\lambda_{k}\right\rangle\left\langle\lambda_{k}\right|
$$

Proof. This is a special case of Lemma 2.1 with a Hermite matrix. Indeed, writing

$$
A=U D U^{*}=\sum_{k=1}^{n} \lambda_{k} U E_{k k} U^{*},
$$

we see that this amounts to (139).

We may directly prove this by operating $A$ resp. $\sum_{k=1}^{n} \lambda_{k} P_{k}$ on an arbitrary $|a\rangle=\sum_{\ell=1}^{n} a_{\ell}\left|\lambda_{\ell}\right\rangle$. We have

$$
A|a\rangle=\sum_{k=1}^{n} a_{k} A\left|\lambda_{k}\right\rangle=\sum_{k=1}^{n} a_{k} \lambda_{k}\left|\lambda_{k}\right\rangle
$$

and

$$
\sum_{k=1}^{n} \lambda_{k} P_{k}|a\rangle=\sum_{k=1}^{n} \lambda_{k}\left|\lambda_{k}\right\rangle\left\langle\lambda_{k}\left|\sum_{\ell=1}^{n} a_{\ell}\right| \lambda_{\ell}\right\rangle=\sum_{k, \ell} a_{\ell} \lambda_{k}\left|\lambda_{k}\right\rangle\left\langle\lambda_{k} \mid \lambda_{\ell}\right\rangle=\sum_{k=1}^{n} a_{k} \lambda_{k}\left|\lambda_{k}\right\rangle
$$

by orthogonality, whence (139) follows.

Proposition 4.2: Let

$$
\sigma_{y}=\sigma_{2}=\left(\begin{array}{cc}
0 & -i \\
i & 0
\end{array}\right)
$$

be one of three three Pauli matrices. Then prove that

$$
e^{i \gamma \sigma_{y}}=\left(\begin{array}{cc}
\cos \gamma & -\sin \gamma \\
\sin \gamma & \cos \gamma
\end{array}\right)
$$

which is the matrix of rotation by $\gamma$

Proof. It is easy to see that the eigenvalues and the corresponding eigenspaces of $\sigma_{y}$ are $\lambda_{1}=1, \lambda_{2}=-1$ , $\quad E_{\sigma_{y}}(1)=\mathbb{C}\left(\begin{array}{c}-i \\ 1\end{array}\right), \quad E_{\sigma_{y}}(-1)=\mathbb{C}\left(\begin{array}{l}i \\ 1\end{array}\right)$. With $\left|\lambda_{1}\right\rangle=\frac{1}{\sqrt{2}}\left(\begin{array}{c}-i \\ 1\end{array}\right)$, $\left|\lambda_{2}\right\rangle=\frac{1}{\sqrt{2}}\left(\begin{array}{l}i \\ 1\end{array}\right)$, we have

$$
\left|\lambda_{1}\right\rangle\left\langle\lambda_{1}\left|=\frac{1}{2}\left(\begin{array}{cc}
1 & -i \\
i & 1
\end{array}\right), \quad\right| \lambda_{2}\right\rangle\left\langle\lambda_{2}\right|=\frac{1}{2}\left(\begin{array}{cc}
1 & i \\
-i & 1
\end{array}\right) .
$$

Substituting this in (141), we conclude (144).

Information processing (IP) $f$ is a process by which an input data is processed by $f$ and an output data is released. This has been done by classical computers whose circuits consist of wires and logical gates, where the former carry information around the circuit and the latter perform manipulations of information from one form to another. A Parallel Information Processing (PIP) is the case where there are several processors $f_{i}$ working on an input to give an output, where processors are not necessarily independent. Quantum Information Processing (QIP) is similar in spirit to PIP in that instead of several processors one uses only one processor-a (programmed) unitary operator as in (42) and the logical gate performing this operation of multiplying by a unitary matrix is called a quantum gate:

$$
\left|\Psi_{\mathrm{out}}\right\rangle=U_{f}\left|\Psi_{\mathrm{i} n}\right\rangle, \quad U_{f}=e^{-\frac{i}{\hbar} H_{f} t},
$$

where $H_{f}$ is the Hamiltonian corresponding to the processor $f$. The quantum computer to be defined in Definition 7 drastically reduces the number of computational steps by means of the generated entanglement.

It is noticeable that quantum physics, esp. quantum entanglement has a close connection with the Riemann zetafunction, esp. the Riemann-Siegel formula [27-31] etc. This will be studied elsewhere.

Example 4.2: The NOT gate acting on single qubits is the one which changes $|0\rangle$ and $|1\rangle$ and can be represented by the matrix

$$
X=\left(\begin{array}{ll}
0 & 1 \\
1 & 0
\end{array}\right)
$$

which is a unitary matrix and one of Pauli matrices denoted $\sigma_{1}=\sigma_{x}=X$.

Let $U$ be a unitary matrix of degree2. Then the controlled $U$-gate acting on 2-qubits is the one which transforms the second bit (target bit) by $U$ only when the first bit (the control bit) is $|1\rangle$. In the special case where the unitary matrix $U$ is given by

$$
U_{\mathrm{CNOT}}=\left(\begin{array}{llll}
1 & 0 & 0 & 0 \\
0 & 1 & 0 & 0 \\
0 & 0 & 0 & 1 \\
0 & 0 & 1 & 0
\end{array}\right)=\left(\begin{array}{cc}
E_{2} & O \\
O & X
\end{array}\right),
$$

the controlled $U$-gate is called the CNOT (controlled NOT gate). I.e. CNOT gate acts on 2-qubits and changes $|x y\rangle$ into $|x(x+y) \bmod 2\rangle$, i.e. it changes the target bit-the second bit by the other one only when the control bit-the first bit is 1 .

For any unitary matrices used $U_{1}, U_{2}$, the conditional transformation

$$
U=|0\rangle\left\langle 0\left|\otimes U_{1}+\right| 1\right\rangle\langle 1| \otimes U_{2}
$$

is also unitary. For

$$
U U^{*}=\left(|0\rangle\left\langle 0\left|\otimes U_{1}+\right| 1\right\rangle\langle 1| \otimes U_{2}\right)\left(|0\rangle\left\langle 0\left|\otimes U_{1}^{*}+\right| 1\right\rangle\langle 1| \otimes U_{2}^{*}\right)
$$

reduces to $|0\rangle\left\langle 0\left|\otimes E_{2}+\right| 1\right\rangle\langle 1| \otimes E_{2}=E_{2} \otimes E_{2}=E_{4}$ since

$$
E_{i i} E_{j j}=\delta_{i j} E_{i j}
$$


Since

$\left(\begin{array}{cc}E_{2} & O \\ O & X\end{array}\right)=\left(\begin{array}{cc}E_{2} & O \\ O & O\end{array}\right)+\left(\begin{array}{cc}O & O \\ O & X\end{array}\right)=E_{11} \otimes E_{2}+E_{22} \otimes X=|0\rangle\left\langle 0\left|\otimes E_{2}+\right| 1\right\rangle\langle 1| \otimes X$,

it follows that the matrix $U_{\mathrm{CNOT}}$ in (148) is a conditional transformation and a fortiori is a unitary matrix.

Example 4.3: The Hadamard gate $H$ is defined by

$$
H|x\rangle=\frac{1}{\sqrt{2}} \sum_{y=0}^{1}(-1)^{x y}|y\rangle .
$$

Hence it sends $|0\rangle$ (resp. $|1\rangle$ ) to $\frac{1}{\sqrt{2}}(|0\rangle+|1\rangle$ ) (resp. $\left.\frac{1}{\sqrt{2}}(|0\rangle-|1\rangle)\right)$ and is represented by the matrix $H=\frac{1}{\sqrt{2}}\left(\begin{array}{cc}1 & 1 \\ 1 & -1\end{array}\right)$ . Since $H^{2}=E_{2}$ we have

$$
H H|0\rangle=|0\rangle, \quad H H|1\rangle=|1\rangle .
$$

Consider the CNOT gate with the control and target bits are squeezed by two Hadamard gates. Then if the first input bit is $|1\rangle$, then by the conditional negation, the second input bit is interchanged, i.e. the second input bit after the controlled negation becomes $H|1\rangle$ (resp. $H|0\rangle$ ) if the second input bit is $H|0\rangle$ (resp. $H|1\rangle$ ). Then this is processed by the last Hadamard gate to become $H|1\rangle$ (resp. $H|0\rangle$ ), i.e. this circuit has the same effect as the up-side-down CNOT gate.

We have also

$$
H \otimes H|00\rangle=H \otimes H|0\rangle \otimes 0\rangle=H|0\rangle \otimes H|0\rangle=\frac{1}{\sqrt{2}}(|0\rangle+|1\rangle) \otimes \frac{1}{\sqrt{2}}(|0\rangle+|1\rangle) .
$$

Let

$$
U=\left(\begin{array}{cc}
E_{2} & O \\
O & \tilde{U}
\end{array}\right)
$$

where

$$
\tilde{U}=\left(\begin{array}{ccc}
a & \circ & b \\
\circ & E_{4} & \circ \\
c & \circ & d
\end{array}\right)
$$

where $a, b, c, d \in \mathbb{C}$ satisfy the conditions under which $\tilde{U}$ is unitary. Let

$$
\tilde{\tilde{U}}=\left(\begin{array}{ll}
a & b \\
c & d
\end{array}\right)
$$

$U$ acts non-trivially only on the 3rd qubit $\alpha|010\rangle$ and the 8 th q-bit $\beta|111\rangle$ of a 3-qubit giving rise to

$$
{ }^{t}(0,0, a \alpha+b \beta, 0,0,0,0, c \alpha+d \beta) .
$$

We interpret this as an action of $\tilde{U}$. A Grey code connecting $|010\rangle$ and $|111\rangle$ is

$$
g_{1}=|010\rangle \rightarrow g_{2}=|011\rangle \rightarrow g_{3}=|111\rangle \text {. }
$$

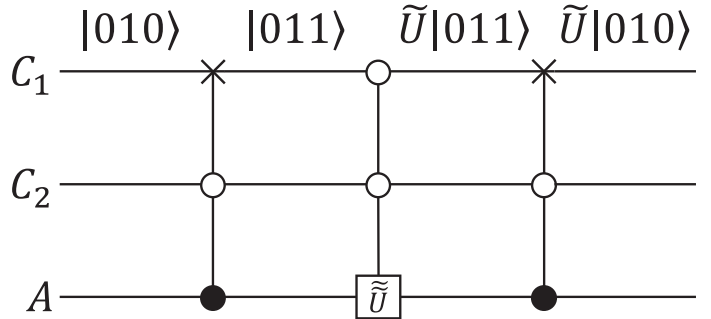

Figure 1: Circuit implementing $U$ in (155).

Since $\mathrm{g}_{2}$ and $\mathrm{g}_{3}$ are different only at the first qubit, we think of them as contracted to $\alpha|0\rangle+\beta|1\rangle=\left(\begin{array}{l}\alpha \\ \beta\end{array}\right)$ and multiply it by $\tilde{\tilde{U}}$ and then pull back the first entry $a \alpha+b \beta$ to the 3rd qubit $|010\rangle$-position as shown in Figure 1.

In Figure 1, $\left.a c_{2} c_{1}\right\rangle$ is input to the gate $A C_{2} C_{1}$. The cross symbol means the NOT gate and so 010$\rangle$ is output as 011$\rangle$. Then after operation by $\tilde{U}, \tilde{U} 011\rangle$ is output as $\tilde{U} 010\rangle$.

It can be shown that any unitary matrix $U$ can be expressed as the product of level 2-matrices. This is a step toward the proof of Theorem 4.3 at the end. For its proof we refer to [25].

The theory of finite Fourier transforms (or discrete Fourier transform) has been developed in [32] in the case of periodic functions and in [17, pp. 109-114] in the case of a finite group. Cf. also [33, 8.1], [22, 4.1, 4.3].

Definition 5: Let

$$
\varepsilon_{j}(x)=e^{2 \pi i x / N}, \quad 1 \leq j \leq N,
$$

where $x$ is an integer variable and $N$ is defined by (119). Then the set $\left\{\varepsilon_{j}(x) \mid 1 \leq j \leq N\right\}$ forms a basis of the vector space $C(N)$ of all periodic arithmetic functions with period $N$, where an arithmetic function is one defined only for integer arguments. We define the discrete Fourier transform (DFT) $\hat{f}$ (or the $y$ th Fourier coefficient) of $f$ by

$$
\hat{f}(y)=\frac{1}{\sqrt{N}} \sum_{x=0}^{N-1} \varepsilon_{y}(-x) f(x) .
$$

Then the Fourier inversion or Fourier expansion formula holds true:

$$
f(x)=\sum_{y=0}^{N-1} \hat{f}(y) \varepsilon_{y}(-x)
$$

Note that (161) is the expression of $f$ with respect to the basis $\left\{\varepsilon_{j}\right\}$.

Example 4.4: The case $N=2$ of (160) amounts to the Hadamard gate. This is so since $\varepsilon_{j}(x)=(-1)^{x j}$ and the quantum gate realizing the DFT $(N=2)$ coincides with (152).

Definition 6: Let 


$$
u_{1}, \cdots, u_{N} \quad\left(N=2^{v}\right)
$$

be an ONB of $H$ and le $\quad|\psi\rangle=\sum_{m=1}^{N} a_{m}\left|u_{m}\right\rangle$,

where $a_{m}=\left\langle\psi \mid u_{m}\right\rangle$. Then we define the measurement operator $M_{m}$ by

$$
M_{m}|\psi\rangle=a_{m}\left|u_{m}\right\rangle
$$

After the measurement by $M_{m}$, the probability that the result involves $\left|u_{m}\right\rangle$ is

$$
\left.p(m)=\left\langle\psi\left|M_{m}^{*} M_{m}\right| \psi\right\rangle=\left(M_{m}|\psi\rangle, M_{m}|\psi\rangle\right)=\left|M_{m}\right| \psi\right\rangle\left.\right|^{2} .
$$

Normalizing the vector in (164), we see that the state after the measurement is to be

$$
\frac{M_{m}|\psi\rangle}{\sqrt{p(m)}}=\frac{a_{m}}{\sqrt{p(m)}}\left|u_{m}\right\rangle
$$

whose right-hand side is sometimes abbreviated as $|m\rangle$ to indicate that the state after the measurement is the $m$ th basis element, so that more information is contained here than (132).

\section{Remark 1 Since}

$$
\left\langle\psi\left|M_{m}^{*}=\right| \psi\right\rangle^{*} M_{m}^{*}=\left(M_{m}|\psi\rangle\right)^{*}=\left(a_{m}\left|u_{m}\right\rangle\right)^{*}=\bar{a}_{m}\left|u_{m}\right\rangle
$$

by (164), it follows that

$$
p(m)=\left|a_{m}\right|^{2}\left\langle u_{m} \mid u_{m}\right\rangle=\left|a_{m}\right|^{2} .
$$

This explains the statement after (113) about the probability of a qubit $a|0\rangle+b|1\rangle$.

(34) is viewed as the probability, which is the inner product $\langle\psi \mid \psi\rangle$. Since (165) is also an inner product $\left\langle M_{m} \psi \mid M_{m} \psi\right\rangle$ by (165), it is reasonable to view (165) as the probability.

Example 4.5: (i) By (127),

$$
M_{1}=M_{|0\rangle}=|0\rangle\left\langle 0\left|, \quad M_{2}=M_{|1\rangle}=\right| 1\right\rangle\langle 1|
$$

work as measurement operators on 1-qubits: If $|\psi\rangle=a|0\rangle+b|1\rangle$ then $p(0)=|a|^{2}, p(1)=|b|^{2}$, so that the state after the measurement (166) reads

$$
\frac{M_{|0\rangle}|\psi\rangle}{|a|}=\frac{a}{|a|}|0\rangle, \quad \frac{M_{|1\rangle}|\psi\rangle}{|b|}=\frac{b}{|b|}|1\rangle .
$$

(ii) Similarly, the measurement operators acting on the first qubit of a 2-qubit given by (136) are

$$
M_{1}=|0\rangle\left\langle 0\left|\otimes E_{2}=\left(\begin{array}{cc}
E_{11} & O \\
O & E_{11}
\end{array}\right), \quad M_{2}=\right| 1\right\rangle\langle 1| \otimes E_{2}=\left(\begin{array}{cc}
E_{22} & O \\
O & E_{22}
\end{array}\right) .
$$

Expressing (136) as

$$
|\psi\rangle=|0\rangle \otimes\left(a_{1}|0\rangle+a_{2}|1\rangle\right)+|1\rangle \otimes\left(a_{3}|0\rangle+a_{4}|1\rangle\right) .
$$

we obtain

$$
\begin{aligned}
& M_{1}|\psi\rangle=\left(|0\rangle\langle 0| \otimes E_{2}\right)\left(|0\rangle \otimes\left(a_{1}|0\rangle+a_{2}|1\rangle\right)\right) \\
& =|0\rangle\langle 0|(|0\rangle) \otimes E_{2}\left(a_{1}|0\rangle+a_{2}|1\rangle\right) \\
& =|0\rangle \otimes\left(a_{1}|0\rangle+a_{2}|1\rangle\right)
\end{aligned}
$$

with $p(0)=\left|a_{1}\right|^{2}+\left|a_{2}\right|^{2}$. Hence the state after the measurement (166) reads

$$
\frac{M_{1}|\psi\rangle}{\sqrt{p(0)}}=|0\rangle \otimes\left(\frac{a}{\sqrt{p(0)}}|0\rangle+\frac{b}{\sqrt{p(0)}}|1\rangle\right)
$$

and similarly for $\frac{M_{1}|\psi\rangle}{\sqrt{p(1)}}$. The measurement operators acting on the second qubit can be introduced verbatim.

Definition 7 Let $H$ be the state space of a quantum system, i.e. the Hilbert space in (116), $U$ be a unitary operator acting on $H$ and let $\left\{M_{m}\right\}$ be a set of measurement operators. The triple (H,U, $\left.\left\{M_{m}\right\}\right)$ is called a quantum computer. In this context, $H$ is called a register and $U$ an algorithm.

Theorem 4.3 (Barenco, et al.) The set of single qubit gates and the CNOT gate is universal, i.e. any quantum circuit or any unitary matrix can be constructed by them.

There are a few well-known quantum algorithms which enable to accomplish computation not possible by conventional computers in polynomial time. For well-known Shor or Glover algorithms, cf. e.g. [25].

Now that the reader has acquired some sense and is to be ready for browsing through the bnewest research papers with less psycological barrier, e.g. [34] and those in the website https://arxiv.org/archive/quant-ph.

\section{References}

1. Li FH, Kanemitsu S, Zhang JJ (2019) From vehicles to grid to electric vehicles to Green Grid, World Sci., London-Singapore-New Jersey 232. Link: https://bit.ly/3rtFJFy

2. Wintner A (1925) Spektraltheorie der unendlichen Matrizen-Einfúhrung in den analytischen Apparat der Quantenmechanik, Hirzel, Leipzig.

3. Romanoff NP (1946) Hilbert spaces and number theory I, Izv. Akad. Nauk SSSR, Ser Mat 10: 3-34. Link: https://bit.ly/3ivijLE 
4. Romanoff NP (1951) Hilbert spaces and number theory II, Izv. Akad. Nauk SSSR, Ser Mat 15: 131-152. Link: https://bit.ly/3exU9PK

5. Feng JW, Kanemitsu S, Kuzumaki T (2021) On Fourier transforms and Hilbert space. Hardy-Ramanujan J 43: 56-68.

6. Chen NX (2010) Möbius inversion in physics. World Sci New Jersey-LondonSingapore etc. Link: https://bit.ly/2Ut1LvV

7. Chebyshev PL (1851) Note sur le differentes séries. J Math Pures Appl 16 337-346. Note on some series, Soč., t. I StPb, 1899, 99-108; Poln. Sobr. Soč Izd. Akad. Nauk SSSR, Moskwa-Leningrad t. 2 (1947), 244-313.

8. Onufrieva LA (1983) The Chebyshev interpolation method in the case of a large number of data. (Russian) Istor.-Mat. Issled. 27: 259-274.

9. Wintner A (1947) An arithmetical approach to ordinary Fourier series, Wavery Press, Baltimore.

10. Kiselev AA, Ozhygova EP (1968) On history of the elementary proof of the prime number theorem, In Actes 11th. Intern. Congr. Histtory Sci. VarsovieKrakovie, Ossolineum, Wroclaw 244-249.

11. Kubota T (1992) Automorphic forms and infinite matrices. Nagoya Math $\mathrm{J}$ 127: 61-82. Link: https://bit.ly/3rnCe36

12. Nielsen MA, Chuang IL (2010) Quantum computation and quantum information, Cambridge UP, Cambridge. Link: https://bit.ly/3iu87Dm

13. Gantmacher FR (1959) The theory of matrices, Book One, Chelsea, New York Link: https://bit.ly/3y57IDv

14. Satake I (1975) Linear algebra, Marcel Dekker, New York. Link: https://bit.ly/3BikHhv

15. Yoshida K (1980) Functional analysis, Springer Verl., Berlin etc.

16. Debnath L, Mikusinski P (1990) Introduction to Hilbert spaces and applications, Academic Press, Boston, etc.

17. Li HL, Li FH, Wang NL, Kanemitsu S (2017) Number theory and its applications II, World Sci., London-Singapore-New Jersey.

18. Yoshida K (1956) Modern analysis, Kyoritsu Shuppan, Tokyo (in Japanese).

19. Pauling L, Wilson EB (1935) Introduction to quantum mechanics, McGraw-Hill, New York. Link: https://bit.ly/3eAq81M
20. Koide S (1971) Quantum theory. 6th ed. Shokabo, Tokyo.

21. Erdélyi A, Magnus W, Oberhettinger F, Tricomi FG (1953) Higher transcendenta functions, Vols I-III, Based, in part, on notes left by Harry Bateman, McGrawHill, New York.

22. Chakraborty K, Kanemitsu S, Tsukada H (2009) Vistas of special functions II, World Sci., New Jersey-London-Singapore etc.

23. Dirac P (1958) The principles of quantum mechanics, 4th ed. Oxford UP Oxford 1958. Link: https://bit.ly/3zj01nB

24. Braunstein SL, Pati AK (2012) Quantum information with continuous variables, Springer Verl., Heidelberg etc.

25. Nakahara M, Ohmi T (2008) Quantum computation-From linear algebra to physical realizations, CRC Press, Boca Raton etc. Link: https://bit.ly/2UjWVkJ

26. Kanemitsu S, Kuzumaki T, Liu JY (2022) Intermediate abstract algebra, to appear.

27. Feiler C, Schreich WP (2013) Entanglement and analytical continuation: an intimate relation told by the Riemann zeta function. New J Phys 15: 063009. Link: https://bit.ly/3rjLSUs

28. Feiler C (2017) Quantum physics and number theory connected by the Riemann zeta function, Thesis, Univ. Ulm.

29. Latorre JI, Sierra J (2015) There is entanglement in primes. Quantum information \& computation archive 15: 622-659.

30. Mack R, Dahl JP, Moya-Cessa H, Stunz WT, Walser R, et al. (2010) Riemann Z from wavepacket dynamics. Phys Rev A 82: 032119. Link: https://bit.ly/3wRMf9Z

31. Torosov BT, Della Valle G, Longhi S (2013) Quantum simulation of the Riemann $\zeta$ function. Phys Rev A 87: 032103. Link: https://bit.ly/36K8Ydy

32. Li F, Li H, Wang NL, Kanemitsu S (2013) Number theory and its applications World Sci., London-Singapore-New Jersey. Link: https://bit.ly/2UruwJr

33. Kanemitsu S, Tsukada H (2007) Vistas of special functions. World Sci New Jersey-London-Singapore etc 228. Link: https://bit.ly/2TnLmsf

34. Wakayama M (2021) Photons and special values of zeta functions. Sugaku Tsushin 25: 24-52.t

Discover a bigger Impact and Visibility of your article publication with Peertechz Publications

\section{Highlights}

* Signatory publisher of ORCID

* Signatory Publisher of DORA (San Francisco Declaration on Research Assessment)

- Articles archived in worlds' renowned service providers such as Portico, CNKI, AGRIS, TDNet, Base (Bielefeld University Library), CrossRef, Scilit, J-Gate etc.

* Journals indexed in ICMJE, SHERPA/ROMEO, Google Scholar etc.

* OAI-PMH (Open Archives Initiative Protocol for Metadata Harvesting)

* Dedicated Editorial Board for every journal

* Accurate and rapid peer-review process

* Increased citations of published articles through promotions

* Reduced timeline for article publication

Submit your articles and experience a new surge in publication services (https://www.peertechz.com/submission).

Peertechz journals wishes everlasting success in your every endeavours.

Copyright: (c) 2021 Yu LW, et al. This is an open-access article distributed under the terms of the Creative Commons Attribution License, which permits unrestricted use, distribution, and reproduction in any medium, provided the original author and source are credited. 